\title{
Use of One and Two Horizontal Plates to Reduce the Drag Force on the Rigid Cylinder Located Inside the Channel: Approach of the Immersed Interface Method
}

\author{
As'ad Alizadeh $^{1,2^{*}}$, Samad Jafarmadar ${ }^{3}$, Hussein Jebrail Zekri ${ }^{2}$ \\ 1 Department of Mechanical Engineering, Urmia University of Technology, Urmia, Iran \\ 2 Department of Mechanical Engineering, College of Engineering, University of Zakho, Zakho City, Iraq \\ 3 Department of Mechanical Engineering, Urmia University, Urmia, Iran \\ * Corresponding author's e-mail: asad.alizadeh2010@gmail.com
}

\begin{abstract}
Immersed interface method is a non-matching boundary approach that has been taken into consideration in recent years. In this method, there is no need to coincide between the fluid and the solid grids. Eulerian grid is used for fluid domain and Lagrangian grid is used for solid domain. Using the Dirac Delta function, the connection between these two grids is established. Separation of the flow from the cylinder surface causes a high pressure drop in some parts of the cylinder, resulting in a dramatic increase in drag force. Drag force reduction is very important in some engineering issues, and several methods have been proposed to achieve this goal. In this study, the flow around a rigid cylinder is simulated. The goal is to reduce the drag force on the cylinder through one and two horizontal plates. The results are in good agreement with prior numerical results.
\end{abstract}

Keywords: rigid cylinder; immersed interface method; numerical simulation; drag force.

\section{INTRODUCTION}

The subject of flow around objects and the phenomenon of vortex shedding caused by that due to practical applications in engineering is of great importance. Among the practical applications of these types of streams, flows around chimneys, high rise buildings, marine structures, suspended bridges, aircraft's wing, ship's propeller, masts, and many more can be mentioned. This type of flow often involves complex phenomena such as flow separation, vortex flow and vortex shedding. The formation of a vortex behind a rigid fixed cylinder is a complex phenomenon, and despite all theoretical and laboratory studies, there are still many unknown aspects. An important advantage of this method is that there is no compulsion to adapt the points of the fluid mesh and the object mesh. Tatsutani et al. studied, numerically and in laboratory, voluminous two-dimensional unsteady flow around two cylinders in a channel with a $20 \%$ obstruction ratio. They examined the effect of the gap between cylinders on flow behavior with Reynolds numbers (Re) between $\mathrm{Re}=200$ and $\mathrm{Re}=1600$ [1]. Sohankar et al. [2] considered the fluid flow around the rectangular cylinders at various angles numerically. Their calculations are made for numbers $\mathrm{Re} \leq 200$ and angles between zero and 90 degrees. They found that the behavior of all flow parameters at angles of less than 20 degrees and more than 70 degrees had a clear difference with other angles. Sohankar [3] presents a numerical study on the non-stationary flow around a cylinder. His calculations were carried out in the range of numbers $R e=45$ to $\mathrm{Re}=200$. Sohankar's results showed that for Reynolds number $\operatorname{Re} \leq 50$ flows behind the cylinder are steady and with increasing Reynolds number takes on an unsteady state. Patnike et al. [4] simulated the flow of a circular cylinder using finite element method. In the range of $\mathrm{Re}=41$ to $R e=200$, they examined the flow through the effects of buoyancy. Bruno et al. [5] investigated the flow around the cylinder with the $\mathrm{Re}=40000$ 
by means of a finite volume dissociation method. While Lee and Bienkiewicz [6] applied a finite element method for simulating a large vortex flow through a cylinder with $\mathrm{Re}=22000$. In this study, we use the Immersed Interface Method (IIM) to simulate the flow around the rigid cylinders. In this method, the effect of the presence of a body immersed in a fluid by adding a force term to the Navier-Stokes equations is considered. $\mathrm{Xu}$ [7] have extended the IIM to the 3D Navier-Stokes equation for simulating fluid-solid interaction. In [8] the no-slip boundary conditions are imposed directly by determining the correct jump conditions for stream function and vorticity. Another Cartesian grid approach has been presented by Ye et al. [9] and Udaykumar et al. [10] using finite volume techniques. Ghafuri et al. [11] simulated the fluid-solid interaction in a barrier microchannel. In their work, the change in membrane hardness is considered to be an important factor in changing hematocrit of the blood. Falavand et al. [12] examined fluid-solid interaction in the shear and poiseuille flow and also examined the effect of the capsule hardness on the flow velocity profile. In this study we use IIM, which is a non-conforming meshes method. The flow around a rigid cylinder is simulated. The goal is to reduce the drag force on the cylinder through one and two horizontal plates.

\section{GOVERNING EQUATIONS}

Navier-Stokes equations for the incompressible flow are as follows:

$$
\nabla \cdot \vec{u}=0
$$

$$
\rho\left(\frac{\partial \vec{u}}{\partial t}+\vec{u} \cdot \nabla \vec{u}\right)=-\nabla p+\eta \nabla^{2} \vec{u}+\vec{F}
$$

where $\vec{u}$ is the fluid velocity,

$p$ is the pressure,

$\rho$ is the density, and

$\mu$ is the viscosity of the fluid. The force

$\vec{F}$ which has the form

$$
\vec{F}(\vec{x}, t)=\int_{\Gamma(t)} f(s, t) \delta(\vec{x}-\vec{X}(s, t)) d s
$$

where $\vec{X}(s, t)$ is the arc-length parameterization of $\Gamma(\mathrm{t})$ is the arc-length, $\vec{x}=(x, y)$ is spatial position, and $f(s, t)$ is the force density. Here, $\delta(\mathrm{x})$ is the Dirac function.

$$
\begin{gathered}
\vec{u}(\vec{X}(s, t), t)=\frac{\partial \vec{X}(s, t)}{\partial t}= \\
=\int_{E} \vec{u}(\vec{x}, t) \delta(\vec{x}-\vec{X}(s, t)) d \vec{x}
\end{gathered}
$$

The force density using an expression of the form,

$$
\vec{f}(\mathrm{~s}, \mathrm{t})=\mathrm{k}\left(\vec{X}^{e}(\mathrm{~s})-\vec{X}(\mathrm{~s}, \mathrm{t})\right)
$$

where $\kappa$ is a constant, $k>>1$, and $\vec{X}^{e}$ is the arc-length parameterization of the required boundary position. The respective normal and tangential components of the force density $f_{1}=\vec{f}(s, t) \cdot \vec{n}$ and $f_{2}=\vec{f}(s, t) \cdot \vec{\tau}$ can be related to the jump conditions for pressure and velocity as follows [13]:

$$
\begin{gathered}
{\left[\mu \vec{u}_{\eta \eta}\right]=\mathrm{\kappa} f_{2} \vec{\tau},} \\
{\left[\mu \vec{u}_{\xi \eta}\right]=-\frac{\partial f_{2}}{\partial \eta} \vec{\tau}-\mathrm{\kappa} f_{2} \vec{n},}
\end{gathered}
$$

$$
\begin{gathered}
{\left[\mu \vec{u}_{\xi \xi}\right]=-\left[\mu \vec{u}_{\eta \eta}\right]+\left[p_{\xi}\right] \vec{n}+\left[p_{\eta}\right] \vec{\tau}+} \\
\rho\left[\vec{u}_{\xi}\right] \vec{u} \cdot \vec{n}
\end{gathered}
$$

We note that from above terms the values of the jumps of the first and second derivatives of velocity and pressure taken with respect to the (x, y)coordinates are achieved by a simple coordinate transformation. We consider the below relations as a simple:

$$
\left[\vec{u}_{x}\right]=\left[\vec{u}_{\xi}\right] n_{1}+\left[\vec{u}_{\eta}\right] \tau_{1}
$$

$$
\left[\vec{u}_{y y}\right]=\left[\vec{u}_{\xi \xi}\right] n_{2}^{2}+2\left[\vec{u}_{\xi \eta}\right] n_{2} \tau_{2}+\left[\vec{u}_{\xi \eta}\right] \tau_{2}^{2}
$$

where $\vec{n}=\left(n_{1}, n_{2}\right)$ and $\vec{\tau}=\left(\tau_{1}, \tau_{2}\right)$ are the Cartesian components of the normal and tangential vectors to the interface at the point considered. 


\section{RESULTS AND DISCUSSION}

Separation of the flow from the cylinder surface causes a high pressure drop in some parts of the cylinder, resulting in a dramatic increase in drag force. Drag force reduction is very important in some engineering issues, and several methods have been proposed to achieve this goal. Among these methods, the use of the plate has been extensively investigated; in these researches, most researchers have focused their attention on reducing drag force by placing the plate at the suction point [14-18]. First, for the validation of the method used, the flow around a cylinder with or without horizontal plates is simulated and compared with the results of Hwang et al. [19]. The results indicate that the use of horizontal plates affects the reduction of drag force on the cylinder. The numerical results show a good agreement with the results of of Hwang et al. [19]. The results for circular cylinders, with and without separator, are given in Table 1 and Figure 1 and Figure $2 . G_{1}$ is the distance between the cylinder and the upstream plate, and $\mathrm{G}_{2}$ is the distance between the cylinder and the downstream plate. The parameters of this article are dimensionless. The drag force is the sum of the frictional and pressure drag force. In fact, the drag force is the sum of the horizontal forces applied by the fluid to the cylinder.

In this paper, the effect of separating plates on the flow around the cylinder as well as the drag coefficient for the ratio of $\mathrm{a} / \mathrm{b}=0.5$ is investigated. $a$ is the smaller diameter and $b$ is the large elliptic diameter. First, the streamlines and vortices around the cylinder is examined without separator, and then the effect of a separator at the upstream on the flow behavior is investigated, and finally, by choosing the optimum value of $G_{1}$ (which produces the lowest drag coefficient), the effect of the second plate at downstream on the flow around the cylinder and the drag coefficient is investigated, and the optimum $G_{2}$ value is determined.

Figure 3 shows the streamlines at $\mathrm{Re}=100$ for elliptical cylinders at the instant of 120 seconds. In Figure $3 \mathrm{a}$ on the right of the elliptical cylinder there are two wake values, one of which is being detached and the other one is growing. The drag coefficient obtained for this cylinder (with no separating plate) is 1.43 . In the following the effect of placing a separating plate in top of flow on flow behavior and drag coefficient is investigated. Initially we attach the separator plate to the cylinder, see Figure $3 \mathrm{~b}$. It is noticeable that the size of the vortexes is reduced, and in this case the drag coefficient is equal to 1.14. Increasing the gap between the cylinder and the separator plate up to $\mathrm{G}_{1}=1$, see Figure 3c, the drag coefficient is equal to 0.99 . By increasing the gap between the separator and the cylinder to $\mathrm{G}_{1}=1.5$, the size of the vortex suddenly decreases dramatically and the size of the drag coefficient reaches its minimum of 0.98 , as shown in Figure $3 \mathrm{~d}$. As the distance increases to $G_{1}=2$, the size of the vortex increases again, and the drag coefficient also increases, and in this case it will be equal to 0.99 . Finally, it is observed that with further increase, the distance between the cylinder and the vortex size separator will be almost constant, and the

Table 1. Comparing Cd the results with the results of Hwang et al. [19] for a cylinder, with and without separating plates.

\begin{tabular}{|c|c|c|c|}
\hline Cases & Hwang et al. [19] & Present & Difference (\%) \\
\hline Cylinder without separating plates & 1.33 & 1.30 & 2.25 \\
\hline Cylinder with a downstream separating plate with $\mathrm{G}_{2} / \mathrm{d}=2.0$ & 1.14 & 1.20 & 5.26 \\
\hline Cylinder with an upstream separating plate with $\mathrm{G}_{1} / \mathrm{d}=2.0$ & 0.96 & 0.92 & 4.17 \\
\hline Cylinder with two separating plates and $\mathrm{G}_{1} / \mathrm{d}=1.5$ and $\mathrm{G}_{2} / \mathrm{d}=2.4$ & 0.93 & 0.91 & 2.15 \\
\hline
\end{tabular}

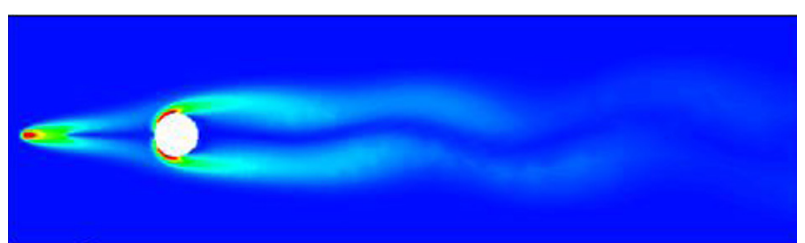

Figure 1. Vortex around and behind the cylinder for cylinder with downstream separating plate with $\mathrm{G}_{1} / \mathrm{d}=2.0$ and $\mathrm{Re}=100$ in the present work. 


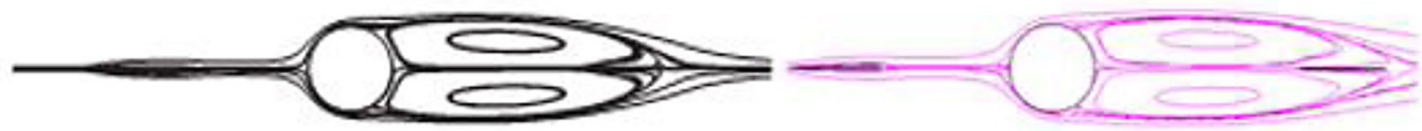

Figure 2. Comparison of streamlines in the present work (on the right) and Hwang et al. [26] (on the left). $\mathrm{Re}=100, \mathrm{G}_{1} / \mathrm{d}=1.5, \mathrm{G}_{2} / \mathrm{d}=2.4, \mathrm{Cd}=0.91$.

drag coefficient will not significantly increase. Figure 4 shows the variation in the drag coefficient $(\mathrm{Cd})$ by the distance of separator relative to the cylinder. As it is shown, the $\mathrm{Cd}$ first shows a decreasing trend so that at $\mathrm{G}_{1}=1.5$ it reaches its minimum value and then it shows an incremental behvior. Thus, for the next step, in which the effect of the size of $\mathrm{G}_{2}$ on the drag coefficient is examined, the second plan is held in place and $\mathrm{G}_{1}$ kept constant, $\mathrm{G}_{1}=1.5$.

In the first case, the value of $\mathrm{G}_{2}$ is zero. By comparing the Figure 5 with Figure $3 \mathrm{~d}$, it is shown that in the absence of a second plane, there is only one wake on the downstream, but by placing the second plate, another wake is also observed. The drag coefficient, which in the case without second plate is 0.98 , in this case its value has dropped to 0.93 . In the next step, the distance $G_{2}$ is set to 0.2 and then to 0.5 . As shown in Figure 5, in

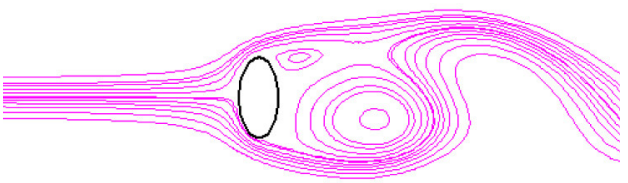

(a)

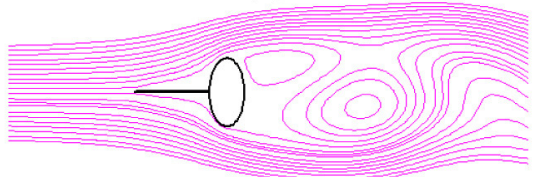

(b)

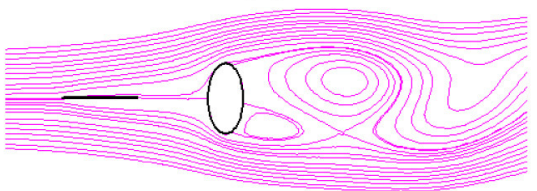

(c)

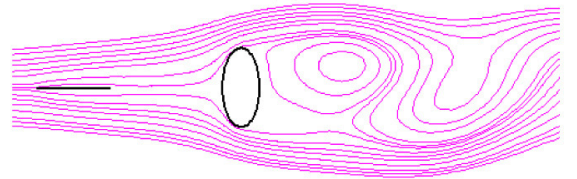

(d)

Figure 3. Stream lines around the cylinder for (a) no-separator plate, (b) $\mathrm{G}_{1}=0$, (c) $\mathrm{G}_{1}=1$, (d) $\mathrm{G}_{1}=1.5$ these cases two wakes are also observed, but the center of the wakes moves away from cylinder compared with previous case, and therefore the size of the drag coefficient has slightly increased, reaching about 0.96 (for $\mathrm{G}_{2}=0.2$ ) and 0.98 (for $\mathrm{G}_{2}=0.5$ ). Then $\mathrm{G}_{2}$ is increased to 1.5 , and it is shown in Figure 5 that one of the wakes get closer to the cylinder and this phenomenon reduces the drag coefficient to about 0.95 . By placing downstream plate away from cylinder, the center of second wake becomes closer to cylinder and this phenomenon causes further decrease of the drag coefficient. The value of the drag coefficient for the $\mathrm{G}_{2}=3, \mathrm{G}_{2}=3.5$ and $\mathrm{G}_{2}=4$ cases are 0.89 , 0.87 and 0.86 , respectively. As the $\mathrm{G}_{2}$ distance increases, the sudden increase in the drag coefficient to about 0.98 has been seen, which is due to the formation of a vortex at the downstream and a decrease in pressure in this region. Figure 6 shows the variation in the drag coefficient $(\mathrm{Cd})$ in terms of the distance of the second separator from the cylinder $\left(G_{2}\right)$. As can be seen, the graph first has an incremental trend, so that at $\mathrm{G}_{2}=0.5$ it reaches its maximum value and then decreases until it reaches its minimum value in $\mathrm{G}_{2}=4.0$ and then increases suddenly at $\mathrm{G}_{2}=4.5$ and for higher values of $\mathrm{G}_{2}$, drag coefficient remains unchanged.

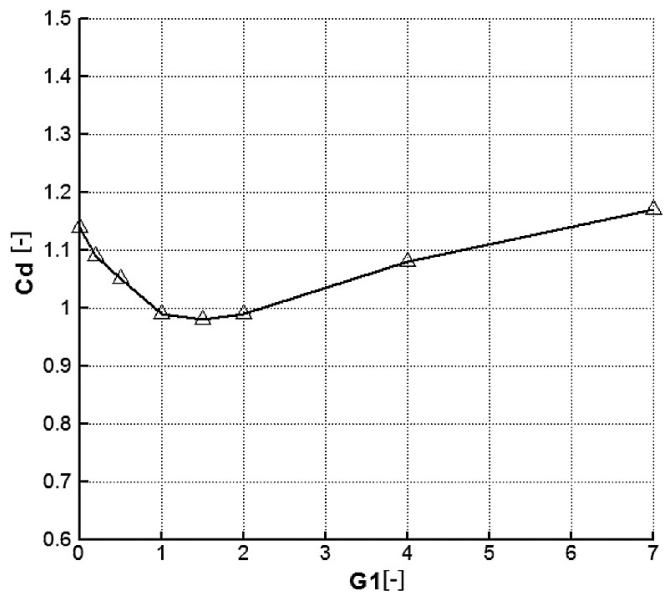

Figure 4. The variation in the Drag coefficient $(\mathrm{Cd})$ in terms of the separator spacing from the cylinder $\left(\mathrm{G}_{1}\right)$ 


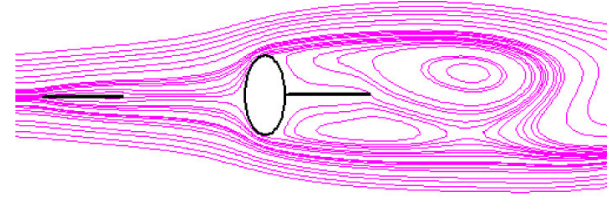

(a)

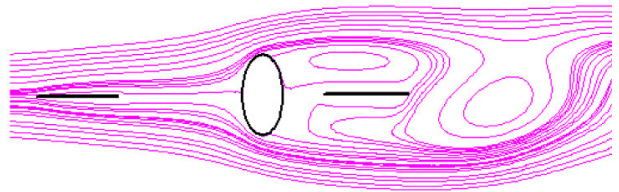

(b)

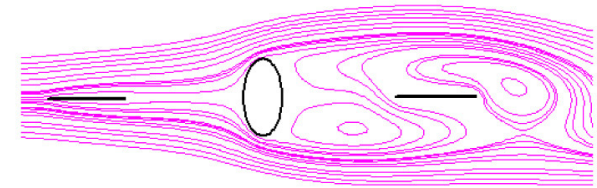

(c)

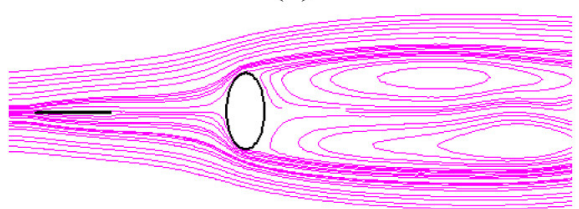

(d)

Figure 5. Stream lines around the cylinder for $\mathrm{G}_{1}=1.5$ and, (a) $\mathrm{G}_{2}=0$, (b) $\mathrm{G}_{2}=0.5$, (c) $\mathrm{G}_{2}=1.5$, (d) $\mathrm{G}_{2}=4$

\section{CONCLUSIONS}

In this study, we have used the immersed interface method, which is a non-conforming meshes method. The flow around a rigid cylinder is simulated. The goal is to reduce the drag force on the cylinder through one and two horizontal plates. The results are in good agreement with other results. First, for the validation of the method used, the flow around a cylinder with or without horizontal plates is simulated and compared with the results of Hwang et al. [19]. The results indicate that the use of horizontal plates affects the reduction of drag force on the cylinder. The numerical results show a good agreement with the results of of Hwang et al. [19].The reduction of drag force on a cylinder is investigated by means of two separated plates from the cylinder which is studied. To do this, two plates of identical length equal to the diameter of the cylinder (d) were placed along the horizontal center line, one on the upstream of the cylinder and the other near the separation zone. Their position is defined by the ratio of intervals $G_{1}$ and $G_{2}$. With changes of these two distance ratios, drag force changes, and in some specific $G$ ratios, for each Reynolds number it has a minimum value. The upstream plate with producing friction reduces the stagnation

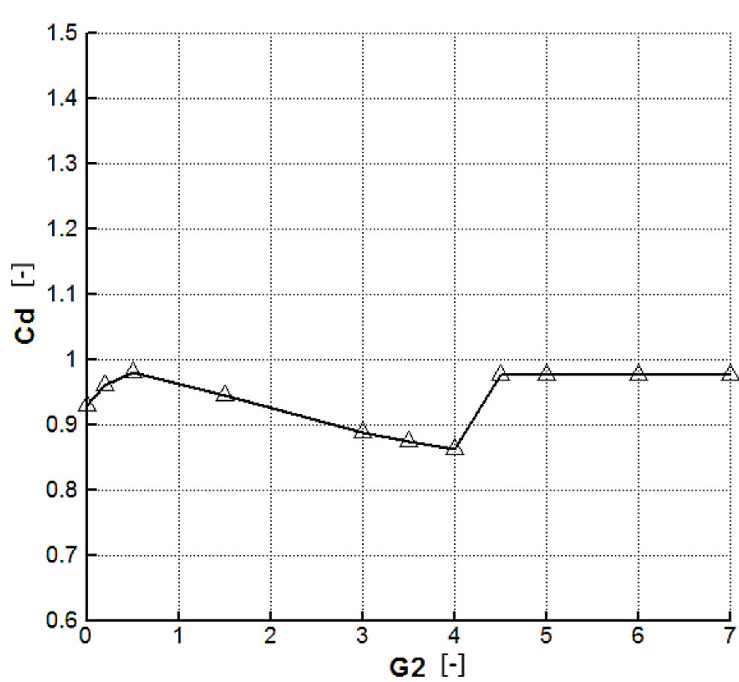

Figure 6. Drag coefficient $\left(\mathrm{C}_{\mathrm{d}}\right)$ variations in terms of the second separator distance from cylinder $\left(\mathrm{G}_{2}\right)$ in case of $\mathrm{G}_{1}=1.5$

pressure, and the downstream plate increases the pressure behind the cylinder by removing vortexes. The combination of these two effects will dramatically decrease drag forces on the cylinder. After passing through the optimal value of $\mathrm{G}_{2}$, the drag force increases sharply, this is due to the formation of vortexes in the wake region.

\section{REFERENCES}

1. Tatsutani, K., Devarakonda, R. and Humphrey, J.A.C. Unsteady flow and heat transfer for cylinder pairs in a channel. International Journal of Heat and Mass Transfer, 36(13), 1993, 3311-3328.

2. Sohankar, A., C. Norbergb, and L. Davidson. Numerical simulation of unsteady low-Reynolds number flow around rectangular cylinders at incidence. Journal of Wind Engineering and Industrial Aerodynamics, 69, 1997, 189-201.

3. Sohankar A. A Numerical Investigation of the Unsteady Wake Flow of Circular Cylinders, 10th Annual International Mechanical Engineering Conference, Tehran, Iran.

4. Patnaik B.S., Narayana P.A. and Seetharamu K.N. Finite element simulation of transient laminar flow past a circular cylinder and two cylinders in tandem-Influence of buoyancy. International Journal of Numerical Methods for Heat \& Fluid Flow, 10(6), 2000, 560-80.

5. Bosco, A., Bruno, L., Coste, N. and Fransos, D. 3D flow around a rectangular cylinder: a computational study. In Proc. BBAA VI Intl. Colloquium on: Bluff Bodies Aerodynamics \& Applications, Milano, Italy, 32, 2008. 
6. Lee, S. and Bogusz B. Finite element implementation of large eddy simulation for separated flows. Journal of Wind Engineering and Industrial Aerodynamics, 77, 1998, 603-617.

7. Xu S. and Wang Z.J. A 3D immersed interface method for fluid-solid interaction, Computer Methods in Applied Mechanics and Engineering. 197, 2008, 2068-2086.

8. Calhoun D. A Cartesian grid method for solving the two-dimensional stream function-vorticity equations in irregular regions. Journal of computational physics. 176, 2002, 231-275.

9. Ye T., Mittal R., Udaykumar H.S. and Shyy W. An accurate Cartesian grid method for viscous incompressible flows with complex immersed boundary, Journal of computational physics. 156, 1999, 209-240.

10. Udaykumar H.S., Mittal R., Rampunggoon P. and Khanna A. A sharp interface Cartesian grid method for simulating flows with complex moving boundaries, Journal of computational physics. 174, 2001, 345-380.

11. Ghafouri A., Esmaily R. and Alizadeh, A. Numerical Simulation of Tank-Treading and Tumbling Motion of Red Blood Cell in the Poiseuille Flow in a Microchannel With and Without Obstacle, Iranian Journal of Science and Technology, Transactions of Mechanical Engineering. 32, 2018, 1-12.

12. Falavand J., Alizadeh A. and Ghafouri A. Modelling of the Dynamics of an immersed body in a microchannel with stenosis using the immersed boundary method. Journal of Computational Applied Mechanics. 43, 2018, 43-52.

13. Abid Mattie, A.; Alizadeh,A.2019. Using a nonconforming meshes method to simulate an interaction between incompressible flow and rigid and elastic boundaries, Mechanika: 25(4) 1-10.

14. Alizadeh,A , Abid Mattie, A.;.2019 Two - dimensional simulation to investigate the interaction of fluid - Structure inside a microchannel with a elastic boundary, International Journal of Mechanical and Production

15. Engineering Research and Development: 9(4) 1151-1156.

16. Rathakrishnan E. Effect of splitter plate on bluff body drag. AIAA Journal. 37(9), 1999, 1125-1126.

17. Unal M.F. and Rockwell D. On vortex formation from a cylinder. Part 2. Control by splitter-plate interference. Journal of Fluid Mechanics. 190, 1988, 513-529.

18. Apelt C.J., West G.S. and Szewczyk A. The effect of wake splitter plates on the flow past a circular cylinder in the range $104<\mathrm{R}<5 \times 104$. Journal of Fluid Mechanics. 61, 1973, 187-198.

19. Kwon K. and Choi H. Control of laminar vortex shedding behind a circular cylinder using splitter plates. Physics of Fluids. 8(2), 1996, 479-486,.

20. Hwang J.Y. and Yang K.S. Drag reduction on a circular cylinder using dual detached splitter plates. Journal of Wind Engineering and Industrial Aerodynamics. 95, 2007, 551-564. 